\title{
On the Use of Passive Reflecting Surfaces and Compressive Sensing Techniques for Detecting Security Threats at Standoff Distances
}

\author{
Yolanda Rodriguez-Vaqueiro and Jose Angel Martinez-Lorenzo \\ ALERT Center of Excellence for the Department of Homeland Security, Gordon CenSSIS, Northeastern University, Boston, MA, USA \\ Correspondence should be addressed to Jose Angel Martinez-Lorenzo; jmartinez@coe.neu.edu
}

Received 28 December 2013; Revised 30 March 2014; Accepted 30 March 2014; Published 2 July 2014

Academic Editor: Tat Soon Yeo

Copyright (C) 2014 Y. Rodriguez-Vaqueiro and J. A. Martinez-Lorenzo. This is an open access article distributed under the Creative Commons Attribution License, which permits unrestricted use, distribution, and reproduction in any medium, provided the original work is properly cited.

\begin{abstract}
This work presents a new radar system concept, capable of detecting explosive related threats at standoff distances. The system consists of a two-dimensional aperture of randomly distributed transmitting/receiving antenna elements and a set of passive reflecting surfaces (PRS) positioned in the vicinity of the target. The PRS act as a mirror that enhances the field of view of the radar system, thus increasing its resolution. A 3D imaging algorithm, based on novel compressive sensing techniques, is used in this work. This system configuration provides a resolution of $7.1 \mathrm{~mm}$ in cross-range and $25 \mathrm{~mm}$ in range, when the target is at $10 \mathrm{~m}$ range, and the radar works at $60 \mathrm{GHz}$ center frequency and has $6 \mathrm{GHz}$ bandwidth.
\end{abstract}

\section{Introduction}

During the last decade, new systems based on MillimeterWave-Radar technology have been deployed in airport checkpoints all around the world [1]. Millimeter-wave imaging systems are preferred to X-ray imaging systems [2-4] for this particular application, because the former do not use ionizing radiation. Millimeter-wave imaging systems have proved to be successful in finding explosives concealed underclothing [5-8]; the success of this technology is mainly due to the short range existing between the sensing components of the system and the person under test. A new important challenge arises when this technology is used for detecting security threats at standoff distances [9-12], which include ranges running between ten to fifty meters. For this particular concept of operation, the resolution of the imaging system is reduced when the range is increased.

Previous work [13] showed a successful 2D reconstruction at $40 \mathrm{~m}$ standoff distance, when a passive array of scatters (PAS) was located between the radar and the person under test. The PAS can be thought of as a millimeter wave lens that enhances the field of view of the radar system, thus increasing its resolution. The same paper also demonstrated that compressive sensing techniques [14-21], which have been successfully used in near-field imaging problems [22,23], can be used for imaging security threats at standoff distances [13].

In this paper, a new type of passive surfaces is used by the imaging system. In particular, the PAS, which works in transmission mode, is replaced by a set of passive reflecting surfaces (PRS), which works in reflection mode. The PRS add additional functionality to the imaging system. Additionally, the imaging algorithm presented in [13] used a single frequency to create 2D images; in this paper, multiple frequencies are used to generate 3D images. Finally, this paper improves the accuracy of the electromagnetic modeling of the passive surfaces by using a physical optics algorithm instead of the ideal point scatter algorithm used in [13].

This paper is structured as follows. Section 2 presents the configuration of the system, Section 3 describes the mathematical formulation for the imaging problem, and Section 4 shows a numerical example. Finally, the last section presents the conclusions.

\section{System Configuration}

2.1. System Concept of Operation. The proposed system configuration is shown schematically in Figure 1. It is composed of an inexpensive, high-resolution radar system that can still 


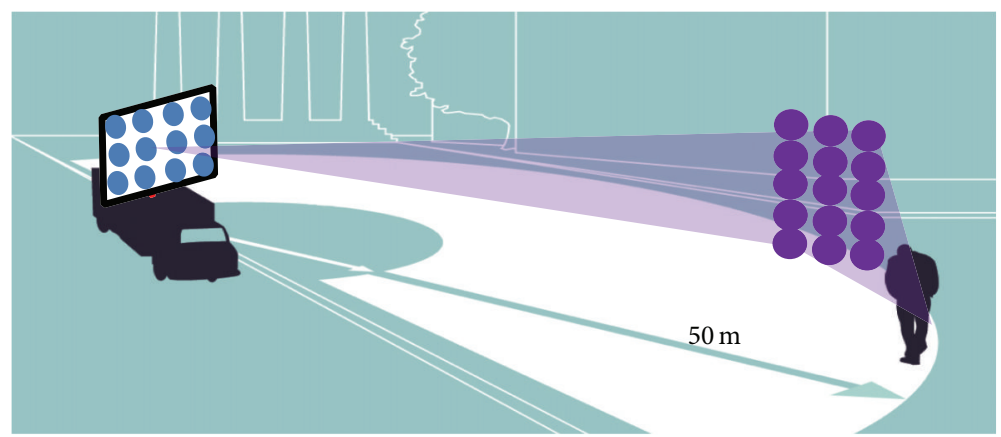

FIGURE 1: General sketch of our van-based, high resolution radar system for standoff detection of potential suicide bombers. The circles in the vicinity of the person under test represent the passive reflecting surfaces used to enhance the field of view and resolution of the imaging system.

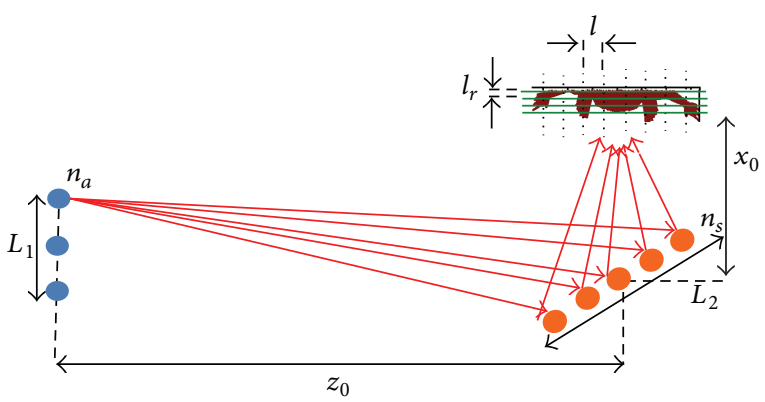

FIGURE 2: Top view of the radar configuration. The blue circles on the left represent a thinned array of transmitter/receiver antennas; the orange dots on the right represent the passive reflecting surfaces, which randomly redirect the energy of the radar towards the target; the person under test (target) is represented by the red silhouette on the top, and the two dimensional planes over which the reconstruction is implemented are represented by the green lines.

fit in or on a van. The field of view of the radar system is increased by using a PRS, which can be thought of as a millimeter wave mirror that enhances the resolution of the imaging system. The PRS are a passive structure that backscatters the electromagnetic waves impinging on its surface; this concept of operation is different than that of the passive array of scatter (PAS) used in [13], which works in transmission mode, acting as a millimeter wave lens, rather than in reflection mode.

2.2. System Parameters. Figure 2 represents a top view of the configuration and the parameters of the system. The blue dots, on the left, represent the positions of the transmitting and receiving antennas. The radar is located on a square aperture of width $L_{1}$, and the total number of transmitting/receiving antennas is $n_{a}$. The orange dots represent the positions of the reflecting elements composing the PRS. The PRS is also located on a square aperture of width $L_{2}$, and the total number of elements on the PRS is $n_{s}$. The person under test is represented by the red silhouette on the top right, and the image reconstruction is performed in $n_{p}$ pixels located on multiple two-dimensional planes, represented by green lines

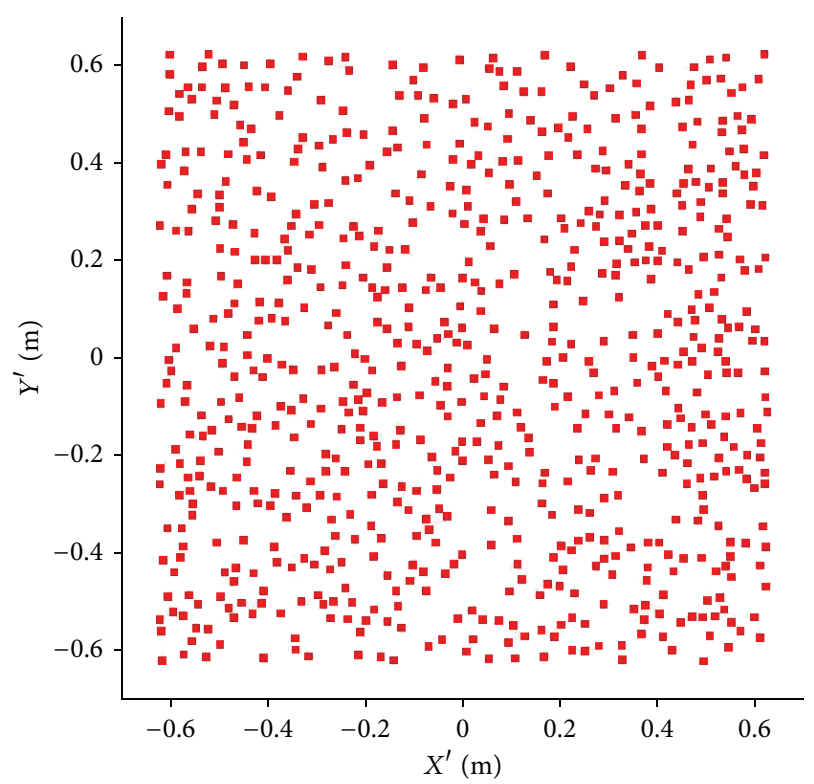

FIGURE 3: Overview of the passive reflecting surfaces (PRS). The variables $X^{\prime}$ and $Y^{\prime}$, expressed in meters, are relative to the center of the PRS.

in Figure 2. The distance between the geometrical center of the radar and the geometrical center of the PAS is $z_{0}$, and the distance between the latter point and the person under test is $x_{0}$. The cross-range resolution $l$ of the imaging system can be approximated by the following equation:

$$
l=\frac{\lambda}{L_{2} \cos \alpha} x_{0}
$$

where $\lambda$ is the wavelength at the center frequency of the imaging system, and $\alpha$ is the angle existing between the normal vector of the plane that contains the PRS and the vector that goes from the center of the PRS to the center of the imaging region. The range resolution $l_{r}$ of the imaging system is given by the following equation [9]:

$$
l_{r}=\frac{c}{2 \mathrm{BW}},
$$




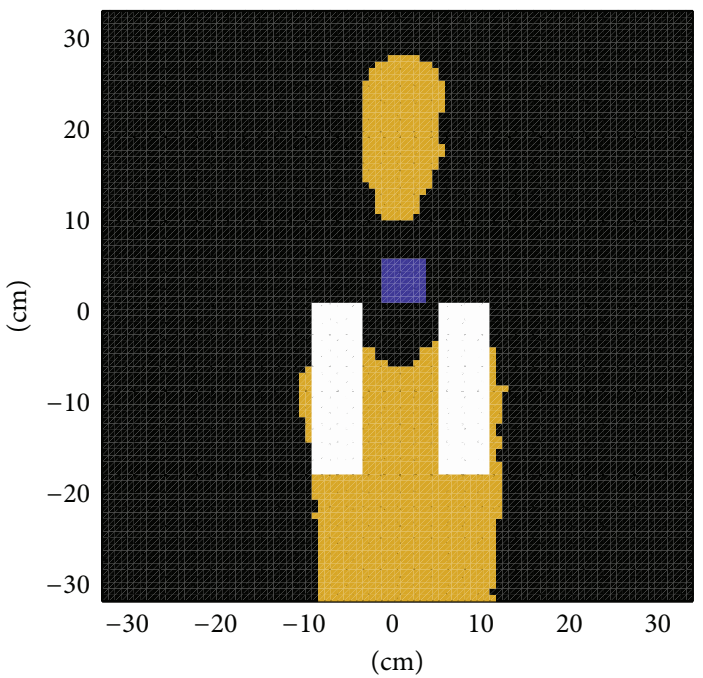

(a)

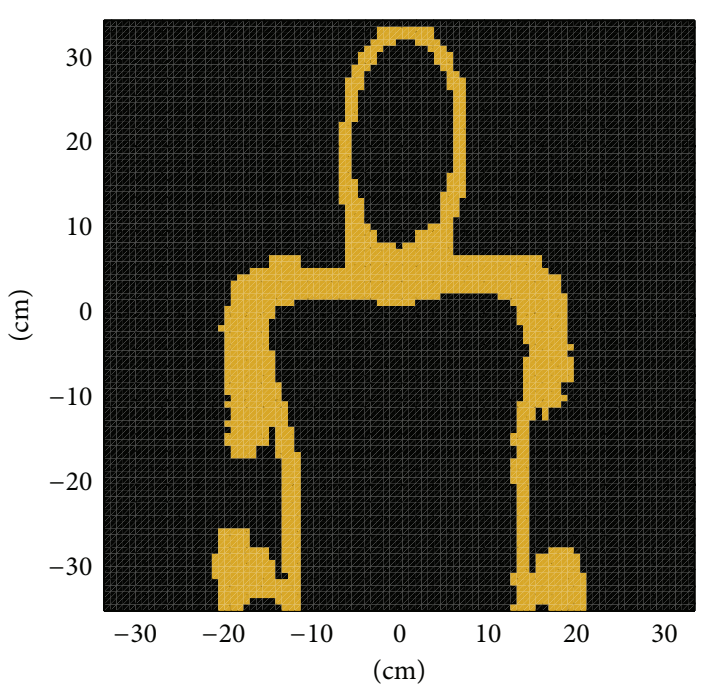

(c)

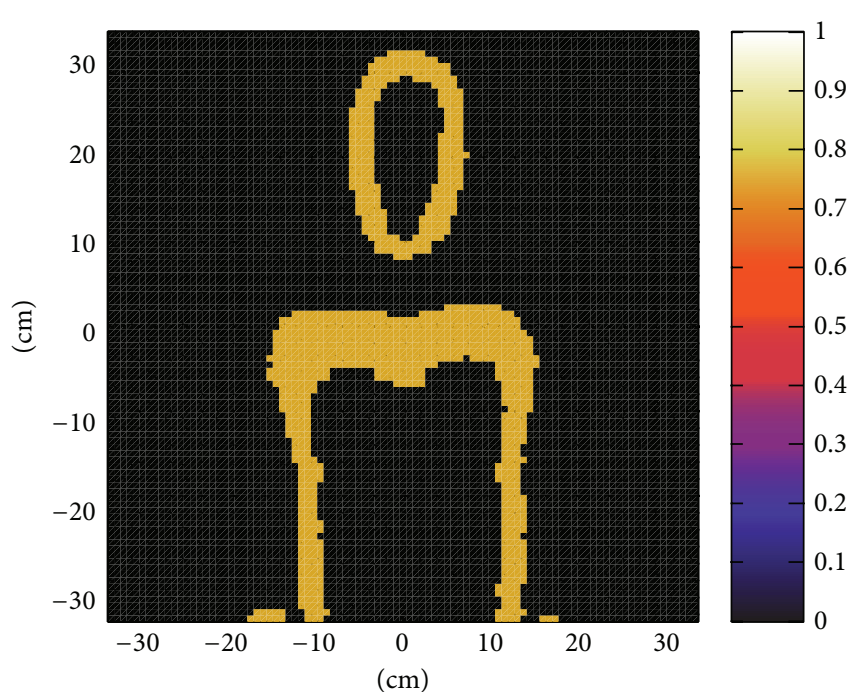

(b)

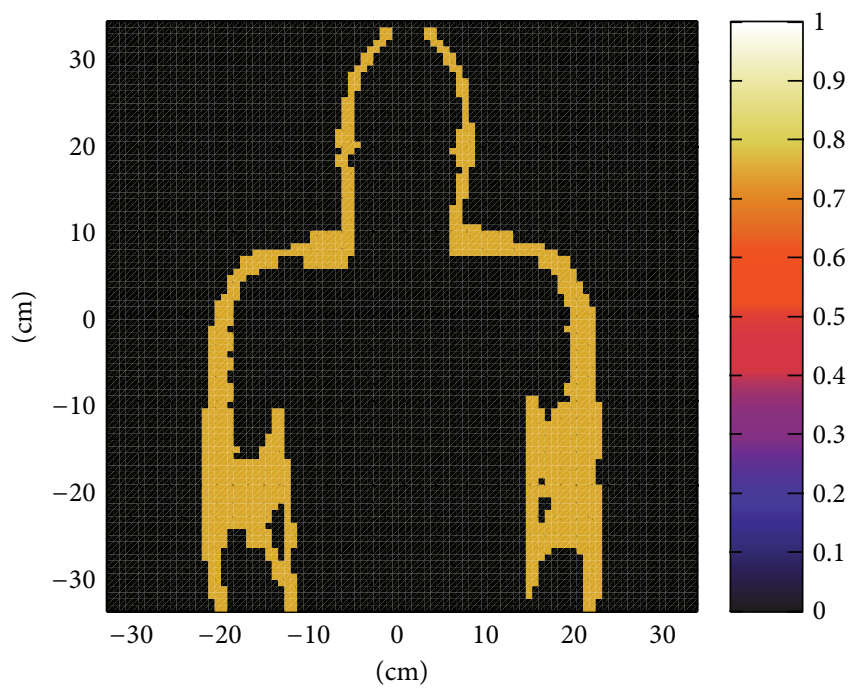

(d)

FIGURE 4: Projected images of the person under test, used as ground truth by the imaging algorithm, at four different ranges: (a) $x_{0}+0$ mm, (b) $x_{0}+30 \mathrm{~mm}$, (c) $x_{0}+60 \mathrm{~mm}$, and (d) $x_{0}+90 \mathrm{~mm}$.

where $c$ is the propagation speed of electromagnetic waves in free space, and BW is the bandwidth of the imaging system. The system uses $n_{f}$ frequencies.

\section{Mathematical Formulation for the Imaging Problem}

3.1. Sensing Matrix. The system works on a multiple monostatic configuration, in which each element of the array transmits and receives in different slots of time without interacting with the radiation of other elements in the array.

Under this configuration, the sensing matrix $A \in C^{n_{t} \times n_{p}}$ establishes a linear relationship between the unknown complex vector $x \in C^{n_{p}}$, with $n_{p}$ the number of unknowns, and the measured complex field data $y \in C^{n_{t}}$, with $n_{t}=n_{a} \cdot n_{f}$, the number of antennas times the number of frequencies. This relationship can be expressed in a matrix form as follows:

$$
A \cdot x+n=y,
$$

where $n \in C^{n_{t}}$ represents the noise collected by each receiving antenna for a given frequency.

Each element $a_{i j}$ of the sensing matrix is computed as follows: (1) the field radiated by the $i$ th antenna, modeled as an infinitesimal aperture [24], is used to illuminate the PRS, for $i=1,2, \ldots, n_{t} ;$ (2) the scattered field produced by the PRS on the imaging region is computed by a physical optics algorithm [25]; (3) the field in the imaging region is backscattered by an infinitesimal sphere located at the center of the $j$ th pixel, for $j=1,2, \ldots, n_{p}$; (4) this backscattered field is used to illuminate the PRS again; and (5) the backscattered field produced by the PRS on the $i$ th antenna 


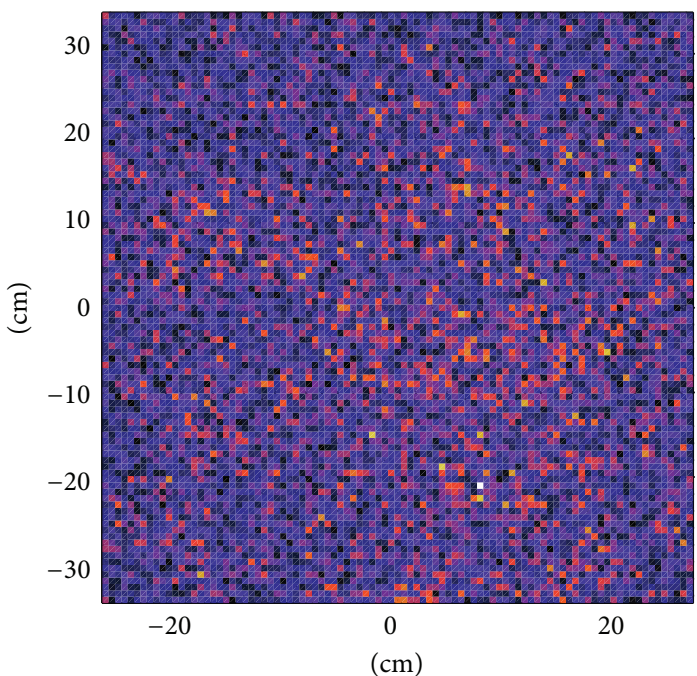

(a)

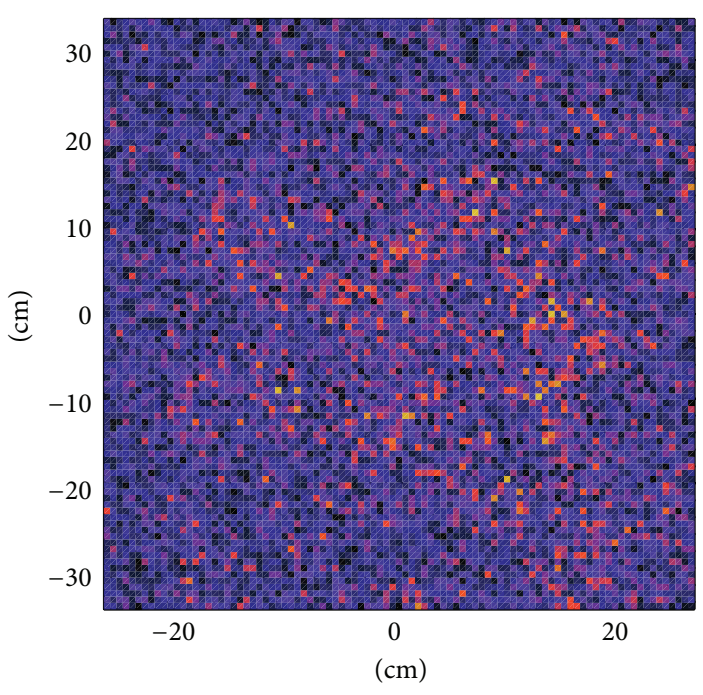

(c)

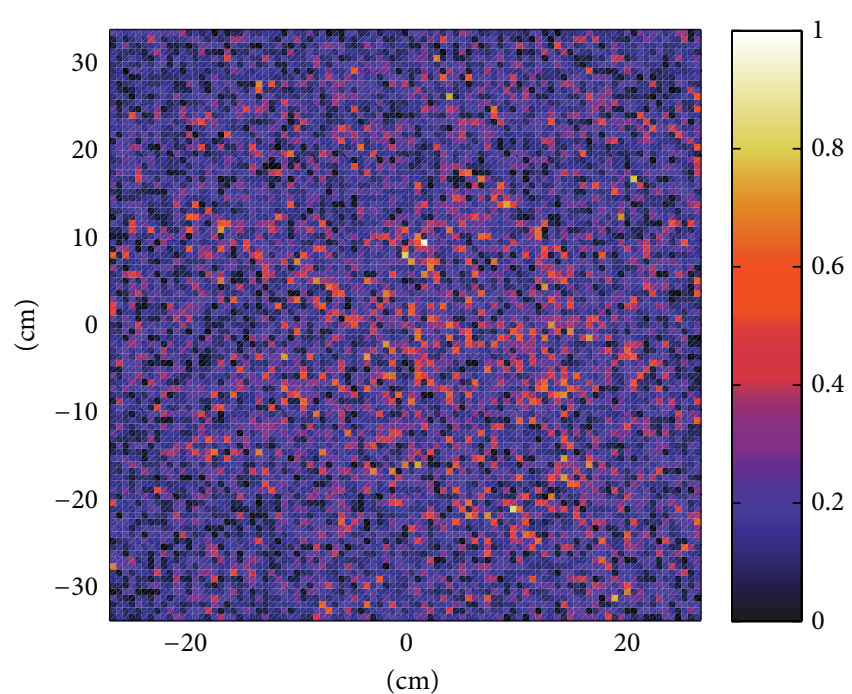

(b)

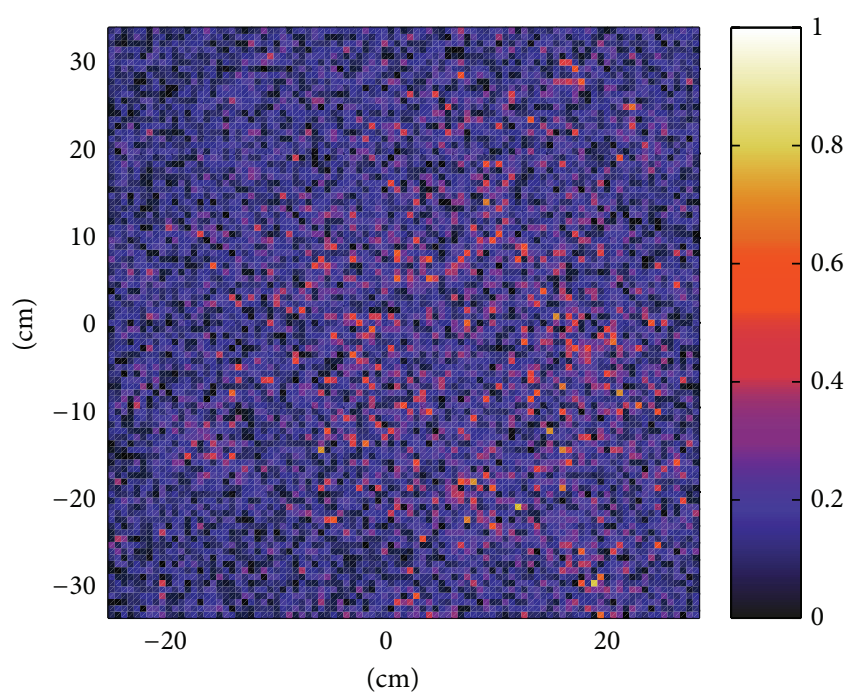

(d)

FIGURE 5: Reconstructed images of the person under test using a traditional SAR algorithm at four different ranges: (a) $x_{0}+0$ mm, (b) $x_{0}+30 \mathrm{~mm}$, (c) $x_{0}+60 \mathrm{~mm}$, and (d) $x_{0}+90 \mathrm{~mm}$.

is stored as the coefficient of the sensing matrix $a_{i j}$. This first order approximation neglects the mutual interaction amongst scatters located in the imaging region.

The PRS are made of metal, and they have fixed reflection coefficient equal to -1 . Having PRS made of different materials, therefore having different reflection coefficient, does not affect to the quality of the reconstructed image if this reflection coefficient is taken into consideration by the sensing matrix.

\subsection{Imaging Algorithm Using Compressive Sensing Approach.} The proposed radar system is designed in accordance with the compressive sensing theory [14-21]. In order to apply such principles for standoff detection of explosive related threats, certain mathematical conditions must be satisfied by the sensing matrix $A$ and the reconstructed reflectivity image $x$. These conditions can be summarized as follows [18]: (1) the sensing matrix must satisfy the restricted-isometry-property condition, which is related to the independence of the columns of the matrix and (2) the unknown reflectivity vector must accept a sparse representation as a solution, which is related to the number of nonzero entries in the solution vector. The parameters of the system can be modified until these two conditions are satisfied; the optimized parameters include the following: aperture length of the radar, aperture length of the PRS, resolution on the reconstruction plane, number of antennas on the radar aperture, number of scatters of the PRS, working frequency, separation between the radar and the PRS, and the separation between the PRS and the target. In this work, this optimization is done manually, but it is expected that in further research contributions such optimization process will be automated. 


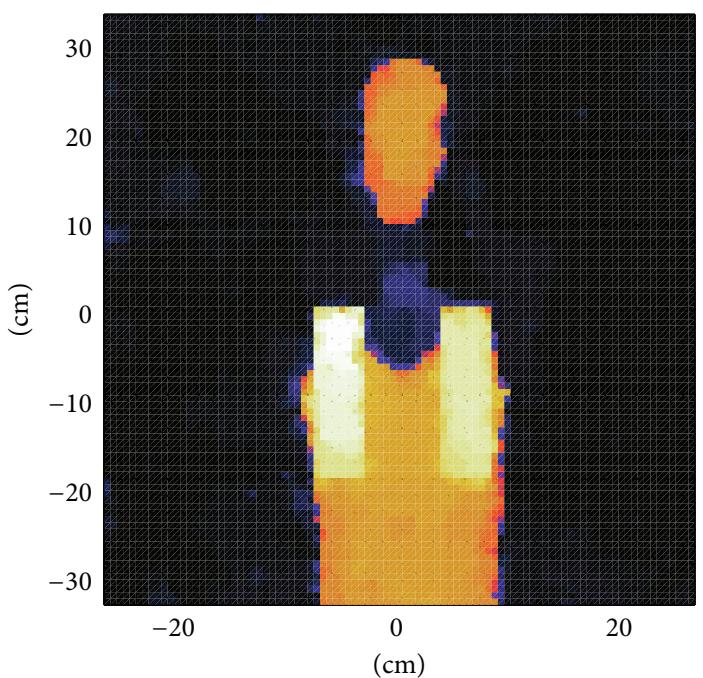

(a)

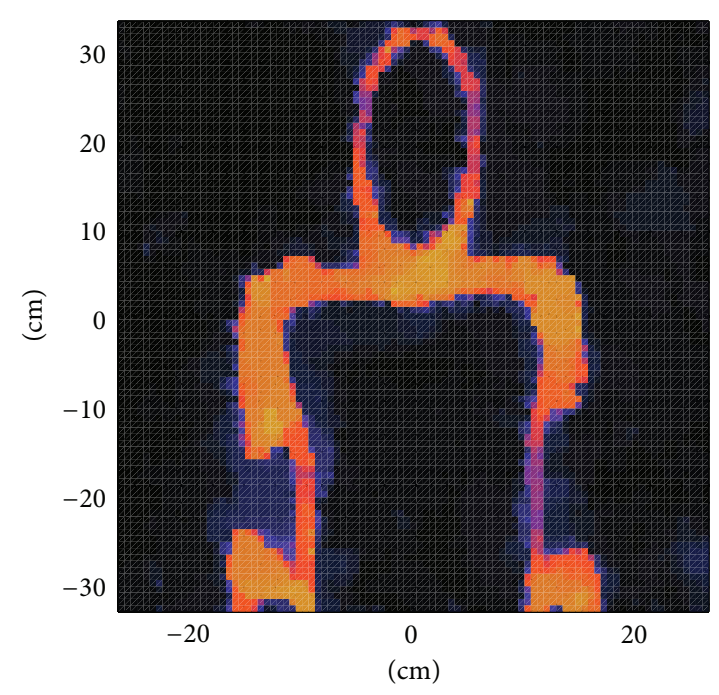

(c)

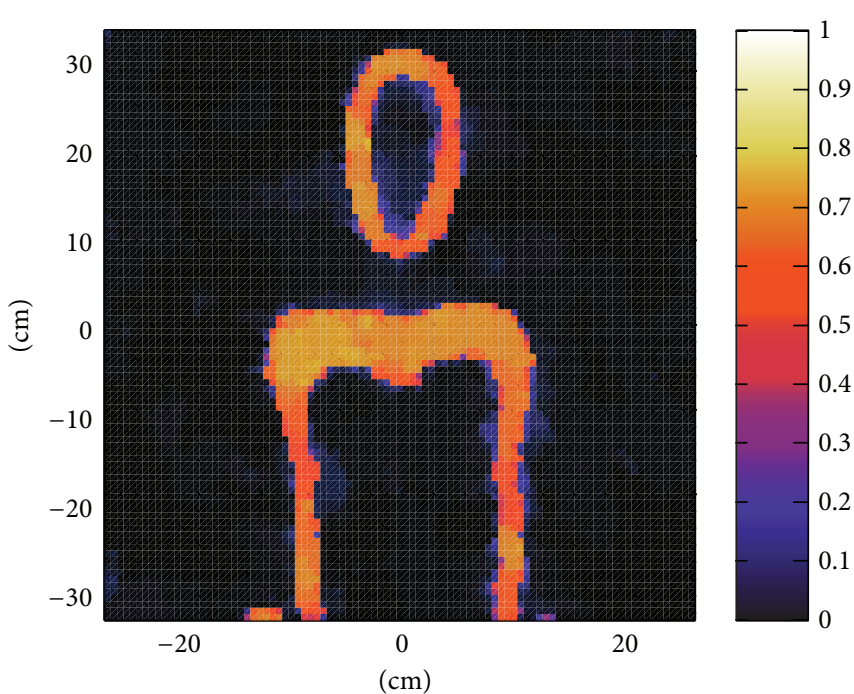

(b)

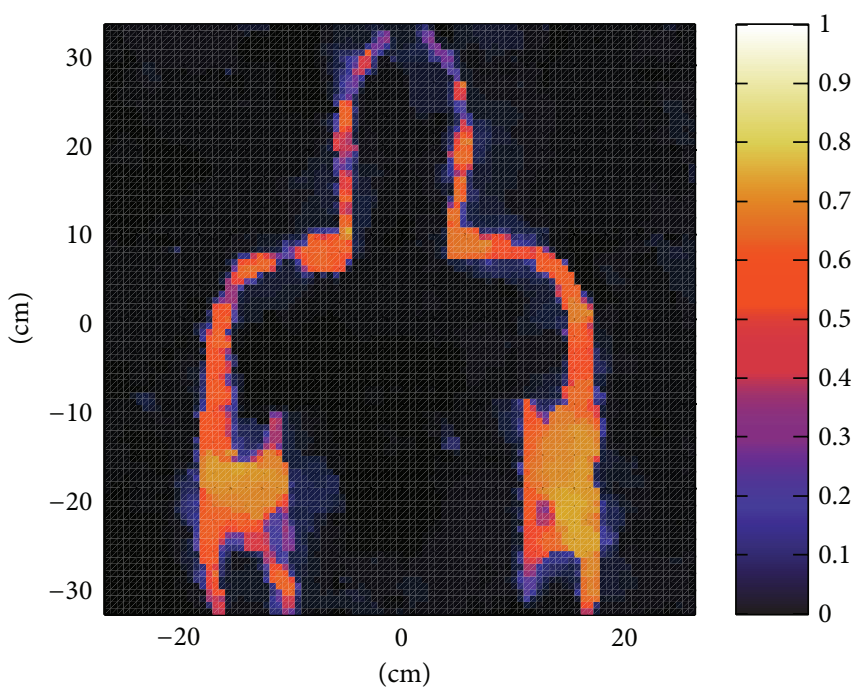

(d)

FIGURE 6: Reconstructed images of the person under test using CS methods at four different ranges: (a) $x_{0}+0 \mathrm{~mm},(\mathrm{~b}) x_{0}+30 \mathrm{~mm}$, (c) $x_{0}+60 \mathrm{~mm}$, and (d) $x_{0}+90 \mathrm{~mm}$.

If the two aforementioned conditions are satisfied, then the reconstruction of the unknown vector can be performed with a small number of measurements (transmitting/receiving antennas) by solving the following convex problem [20]:

$$
\begin{array}{ll}
\min & \|x\|_{1}, \\
\text { s.t. } & \|A x-y\|_{2}<\delta,
\end{array}
$$

where $\|x\|_{1}$ represents the norm-one of the vector $x$, $\|A x-y\|_{2}$ represents the norm-two of the residual error $A x-$ $y$, and $\delta$ is an upper bound for the residual error.

Additionally, it is possible to perform the reconstruction using CS even when the unknown vector is not sparse by using a transformation in a dictionary $W$. The analysis-based problem is expressed as

$$
\begin{array}{ll}
\min & \left\|W^{*} x\right\|_{1}, \\
\text { s.t. } & \|A x-y\|_{2}<\delta,
\end{array}
$$

where $W$ is a dictionary that allows for a sparse representation of $x$, and $(\cdot)^{*}$ is the conjugate operator.

In this particular problem, the magnitude of the gradient of the unknown reflectivity function, which describes the reflectivity properties of the object under test, has been minimized. This transformation of the unknown reflectivity vector $x[k, m, n]$ accepts a sparser representation more than the vector $x[k, m, n]$, where $k$ and $m$ are the cross-range indices and $n$ is the range index of a given pixel in the reconstruction domain. For this reason, a total variation (TV) 


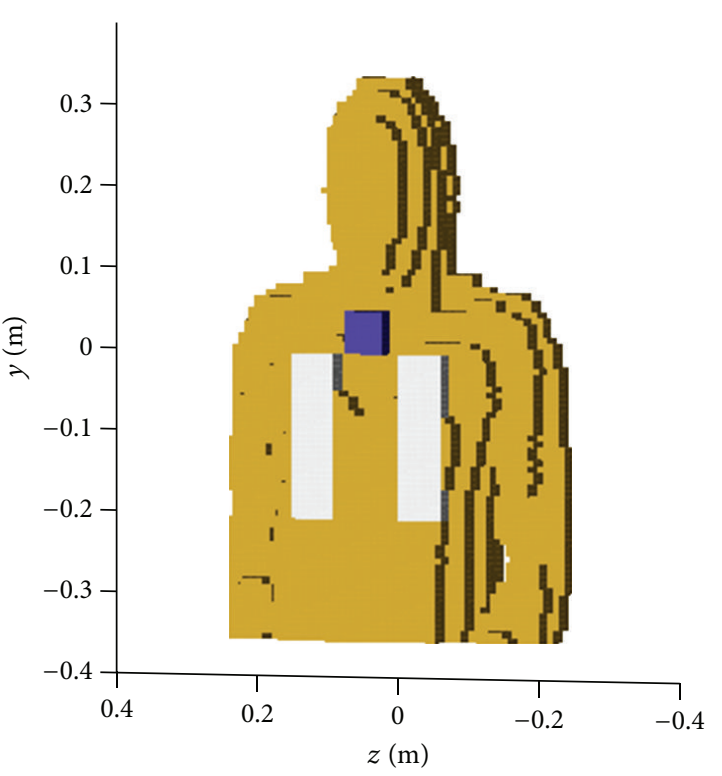

(a)

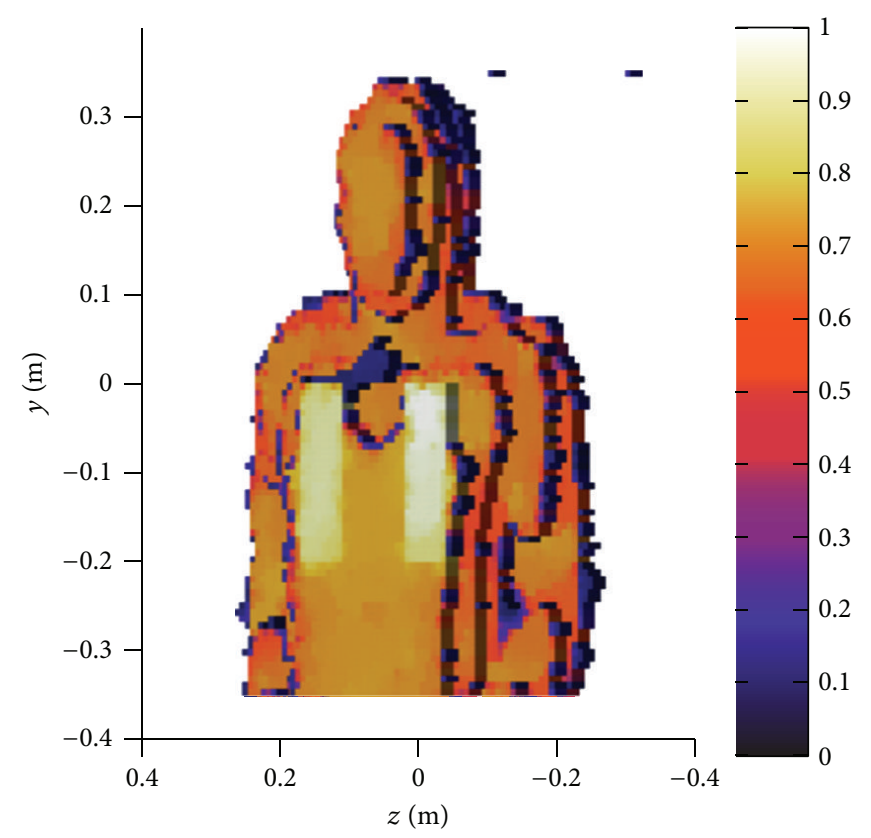

(b)

Figure 7: Three dimensional images created using voxels: (a) ground truth and (b) reconstruction.

transformation is used to reconstruct the image [20]. The TV problem can be written as

$$
\begin{array}{ll}
\min & \|x\|_{\mathrm{TV}}, \\
\text { s.t. } & \|A x-y\|_{2}<\delta,
\end{array}
$$

where $\|x\|_{\mathrm{TV}}$ represents the TV norm of the unknown vector $x$, and it is computed as follows:

$$
\|x\|_{\mathrm{TV}}=\sum_{k, m}\|\nabla x[k, m, n]\|_{2}
$$

where $\nabla$ is given by

$$
\nabla x[k, m, n]=\left[\begin{array}{l}
x[k+1, m, n]-x[k, m, n] \\
x[k, m+1, n]-x[k, m, n]
\end{array}\right] .
$$

It should be noted that $\nabla$ only considers the partial differences in the two cross-range directions. In this way, the reconstruction for each range plane can be performed independently, thus allowing the use of the TV algorithm described in [20].

\section{Results and Discussion}

The performance of the millimeter-wave imaging system is evaluated using a numerical example that simulates security threats at a standoff distance. Table 1 shows the parameters used in the numerical simulation. The total number of measurements used for the reconstruction is given by the number of antennas times the number of frequencies, which is equal to 2340 . The system works in a step frequency configuration, using thirteen uniformly spaced frequencies over a $6 \mathrm{GHz}$ bandwidth centered at $60 \mathrm{GHz}$. The frequency step is chosen so that no aliasing replicas of the image appear in the imaging domain [9]. The PRS is depicted in Figure 3; it is formed by small perfect electric conductor (PEC) facets, which have a flat square shape of side length $3 \lambda=$ $15 \mathrm{~mm}$. Uniform white noise is considered in the simulation, producing a signal to noise ratio of $25 \mathrm{~dB}$.

In this simulation, the person under test carries two types of explosive threats on the chest: (1) two high-reflectivity metallic pipes and (2) one low-reflectivity dielectric box made of TNT. In order to accelerate the speed of the numerical simulation, the reflectivity function of the person under test is projected onto four parallel planes, each one defined at a different range. These projections of the reflectivity function are considered to be the ground truth for the imaging algorithm, and they have been depicted in Figure 4. The colorbar in the image indicates the absolute value of the reflectivity function divided by the average reflectivity of the whole image.

Figure 5 presents the reconstruction obtained using a traditional synthetic aperture radar (SAR) algorithm [9]. Due to the limited number of measurements taken, the recovered image does not provide information about the target.

The reconstructed image of the person under test is shown in Figure 6. The compressive sensing imaging algorithm successfully detects and localizes the security threats on the person's torso. Three dimensional representations of the ground truth and the reconstructed images are shown in Figure 7 , which are also successful in detecting and localizing the security threats.

\section{Conclusions}

This paper describes a millimeter wave imaging system, which is capable of detecting security threats at standoff distances. Unlike traditional imaging systems, in which the radar system directly interrogates the target under test, this 
TABle 1: Parameters for the numerical example.

\begin{tabular}{lc}
\hline Parameter & Configuration \\
\hline$z_{0}$ & $2000 \lambda=8.75 \mathrm{~m}$ \\
$x_{0}$ & $250 \lambda=1.25 \mathrm{~m}$ \\
$L_{1}$ & $80 \lambda=0.4 \mathrm{~m}$ \\
$L_{2}$ & $250 \lambda=1.25 \mathrm{~m}$ \\
$n_{a}$ & 180 \\
$n_{s}$ & 720 \\
$n_{f}$ & 13 \\
$l$ & $7.1 \mathrm{~mm}$ \\
$l_{r}$ & $25 \mathrm{~mm}$ \\
\hline
\end{tabular}

system illuminates passive reflecting surfaces that redirect the energy of the radar towards the person under test. The passive reflecting surfaces act as a millimeter wave mirror that increases the field of view of the imaging system, thus increasing its resolution.

Novel compressive sensing techniques have been used in order to reduce the amount of data collected by the system. The imaging algorithm is based on minimizing the total variation norm, which provides a quick iterative reconstruction of the person under test, thus improving traditional, noniterative, Fourier-based imaging algorithms.

This system configuration provides a resolution of $7.1 \mathrm{~mm}$ in cross-range and $25 \mathrm{~mm}$ in range, and it is capable of producing 3D images.

\section{Conflict of Interests}

The authors declare that there is no conflict of interests regarding the publication of this paper.

\section{Acknowledgments}

This material is based on work supported by the Science and Technology Directorate, U.S. Department of Homeland Security, Award no. "2013-ST-061-ED0001." The views and conclusions contained in this document are those of the authors and should not be interpreted as necessarily representing the official policies, either expressed or implied, of DHS.

\section{References}

[1] D. M. Sheen, D. L. McMakin, and T. E. Hall, “Three-dimensional millimeter-wave imaging for concealed weapon detection," IEEE Transactions on Microwave Theory and Techniques, vol. 49, no. 9, pp. 1581-1592, 2001.

[2] R. F. Eilbert and S. Shi, "Improved imaging for X-ray inspection systems," IEEE Aerospace and Electronic Systems Magazine, vol. 20, no. 3, pp. 23-28, 2005.

[3] S. W. Smith, "X-ray backscatter detection system," US Patent 5, 181, 234, 1993.

[4] TSA, Tsa X-Ray Screening Technology Safety Reports, 2011, http://www.tsa.gov/research/reading/xray_screening_technology _safety_reports_march_2011.shtm.
[5] Y. Álvarez, B. Gonzalez-Valdés, J. A. Martinez, F. Las-Heras, and C. M. Rappaport, "3D whole body imaging for detecting explosive-related threats," IEEE Transactions on Antennas and Propagation, vol. 60, no. 9, pp. 4453-4458, 2012.

[6] Y. Álvarez, B. Gonzalez-Valdés, J. Á. Martinez, F. Las-Heras, and C. M. Rappaport, "An improved SAR based technique for accurate profile reconstruction," IEEE Transactions on Antennas and Propagation, vol. 61, no. 3, pp. 1490-1495, 2013.

[7] B. Gonzalez-Valdés, Y. Álvarez, J. A. Martinez, F. Las-Heras, and C. M. Rappaport, "SAR processing for profile reconstruction and characterization of dielectric objects on the human body surface," Progress in Electromagnetics Research, vol. 138, pp. 269282, 2013.

[8] B. Gonzalez-Valdés, Y. Álvarez, J. A. Martinez, F. Las-Heras, and C. M. Rappaport, "On the use of improved imaging techniques for the development of a multistatic three-dimensional millimeter-wave portal for personnel screening," Progress in Electromagnetics Research, vol. 138, pp. 83-98, 2013.

[9] J. A. Martinez-Lorenzo, F. Quivira, and C. M. Rappaport, "Sar imaging of suicide bombers wearing concealed explosive threats," Progress in Electromagnetics Research, vol. 125, pp. 255272, 2012.

[10] J. Fernandes, C. M. Rappaport, J. A. Martinez-Lorenzo, and M. Hagelen, "Experimental results for standoff detection of concealed body-worn explosives using millimeter-wave radar and limited view ISAR processing," in Proceedings of the IEEE Conference on Technologies for Homeland Security (HST '09), pp. 456-460, May 2009.

[11] A. Angell and C. Rappaport, "Computational modeling analysis of radar scattering by metallic body-worn explosive devices covered with wrinkled clothing," in Proceedings of the IEEE MTT-S International Microwave Symposium (IMS '07), pp. 1943-1946, June 2007.

[12] K. B. Cooper, R. J. Dengler, N. Llombart, B. Thomas, G. Chattopadhyay, and P. H. Siegel, "THz imaging radar for standoff personnel screening," IEEE Transactions on Terahertz Science and Technology, vol. 1, no. 1, pp. 169-182, 2011.

[13] J. A. Martinez-Lorenzo, Y. Rodriguez-Vaqueiro, C. M. Rappaport, O. R. Lopez, and A. G. Pino, "A compressed sensing approach for detection of explosive threats at standoff distances using a passive array of scatters," in Proceedings of the 12th IEEE International Conference on Technologies for Homeland Security (HST '12), pp. 134-139, November 2012.

[14] E. J. Candès, J. Romberg, and T. Tao, "Robust uncertainty principles: exact signal reconstruction from highly incomplete frequency information," IEEE Transactions on Information Theory, vol. 52, no. 2, pp. 489-509, 2006.

[15] E. J. Candès, J. K. Romberg, and T. Tao, "Stable signal recovery from incomplete and inaccurate measurements," Communications on Pure and Applied Mathematics, vol. 59, no. 8, pp. 12071223, 2006.

[16] D. L. Donoho, "Compressed sensing," IEEE Transactions on Information Theory, vol. 52, no. 4, pp. 1289-1306, 2006.

[17] R. G. Baraniuk, "Compressive sensing," IEEE Signal Processing Magazine, vol. 24, no. 4, pp. 118-121, 2007.

[18] A. C. Fannjiang, T. Strohmer, and P. Yan, "Compressed remote sensing of sparse objects," SIAM Journal on Imaging Sciences, vol. 3, no. 3, pp. 595-618, 2010.

[19] M. D. Migliore and D. Pinchera, "Compressed sensing in electromagnetics: theory, applications and perspectives," in Proceedings of the IEEE 5th European Conference on Antennas and Propagation (EUCAP '11), pp. 1969-1973, April 2011. 
[20] S. Becker, J. Bobin, and E. J. Candès, "NESTA: a fast and accurate first-order method for sparse recovery," SIAM Journal on Imaging Sciences, vol. 4, no. 1, pp. 1-39, 2011.

[21] "Nesta: a fast and accurate first-order method for sparse recovery," http://statweb.stanford.edu/ candes/nesta/, 2011.

[22] Y. Rodriguez-Vaqueiro, Y. Álvarez, B. Gonzalez-Valdés, J. Martinez, F. Las-Heras, and C. Rappaport, "On the use of compressed sensing techniques for improving multistatic millimeterwave portal-based personnel screening," IEEE Transactions on Antennas and Propagation, vol. 62, no. 1, pp. 494-499, 2014.

[23] B. Gonzalez-Valdés, G. Allan, Y. Rodriguez-Vaqueiro et al., "Sparse array optimization using simulated annealing and compressed sensing for near-field millimeter wave imaging," IEEE Transactions on Antennas and Propagation, vol. 62, no. 4, pp. 1716-1722.

[24] C. A. Balanis, Advanced Engineering Electromagnetics, vol. 205, Wiley, New York, NY, USA, 1989.

[25] J. A. Martinez Lorenzo, A. G. Pino, I. Vega, M. Arias, and O. Rubiños, "ICARA: induced-current analysis of reflector antennas," IEEE Antennas and Propagation Magazine, vol. 47, no. 2, pp. 92-100, 2005. 

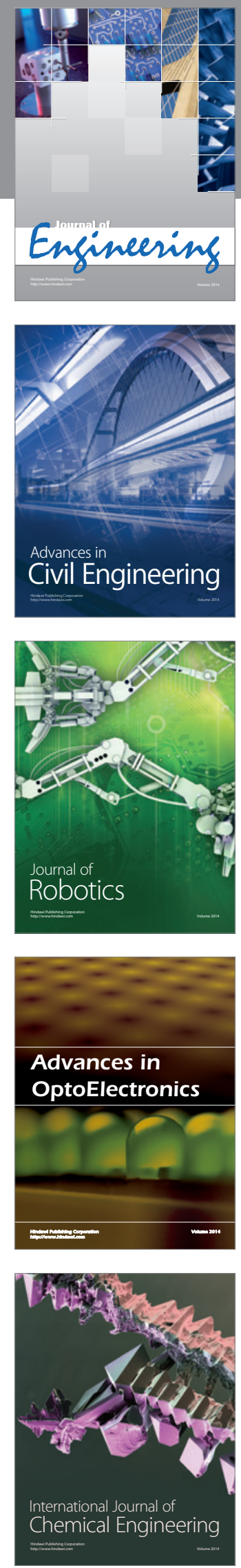

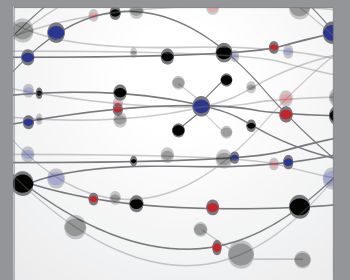

The Scientific World Journal
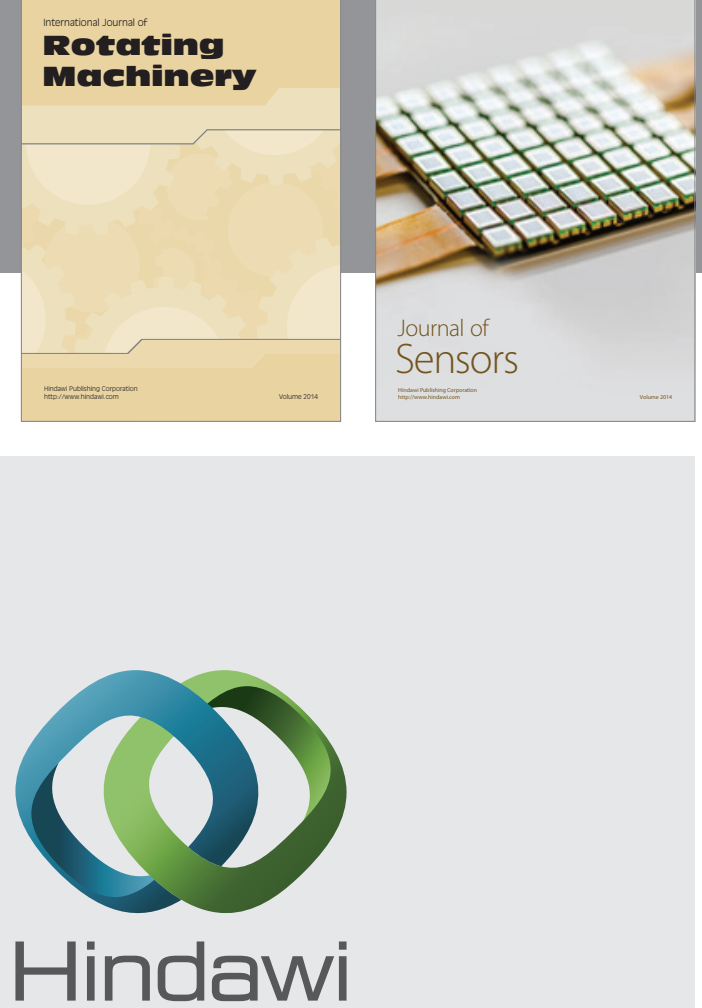

Submit your manuscripts at http://www.hindawi.com
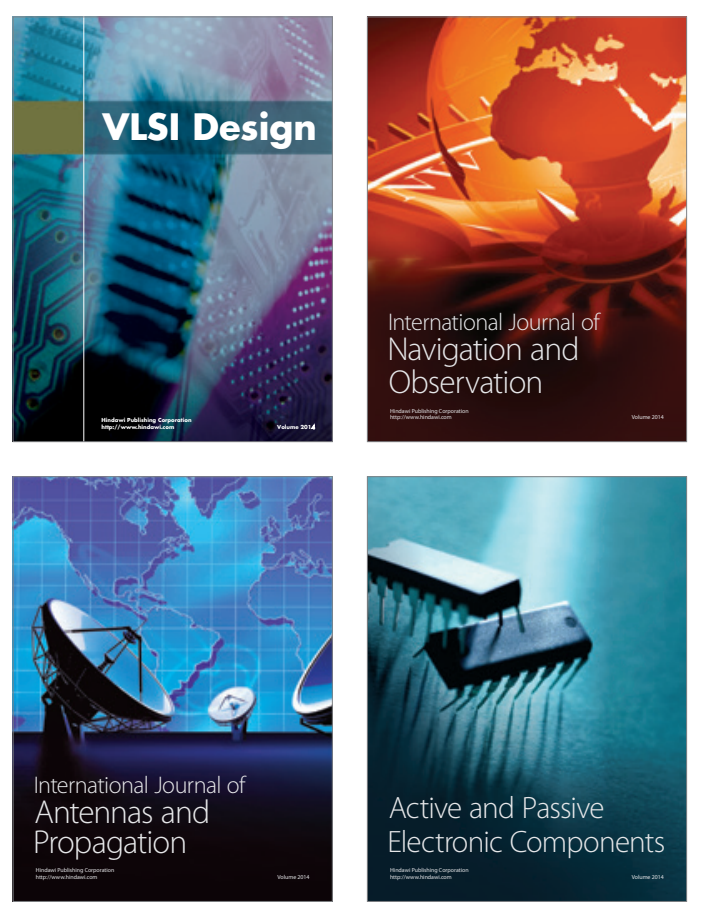
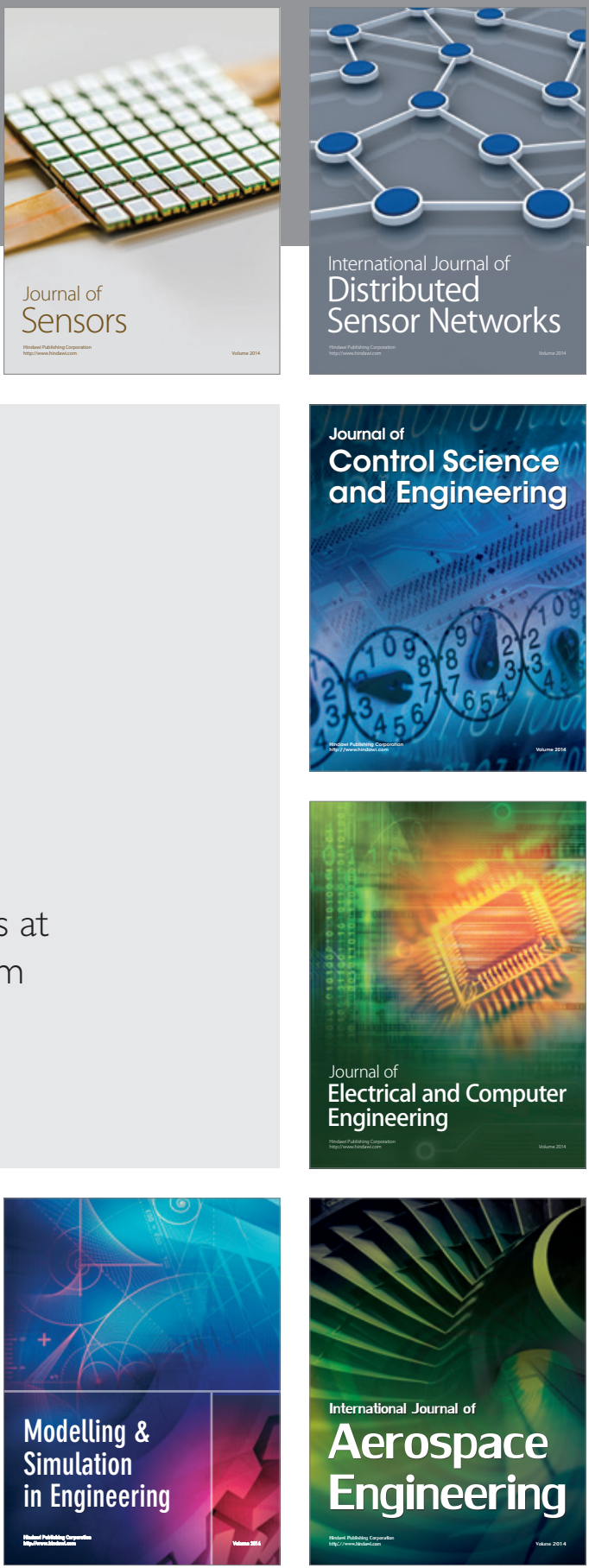

Journal of

Control Science

and Engineering
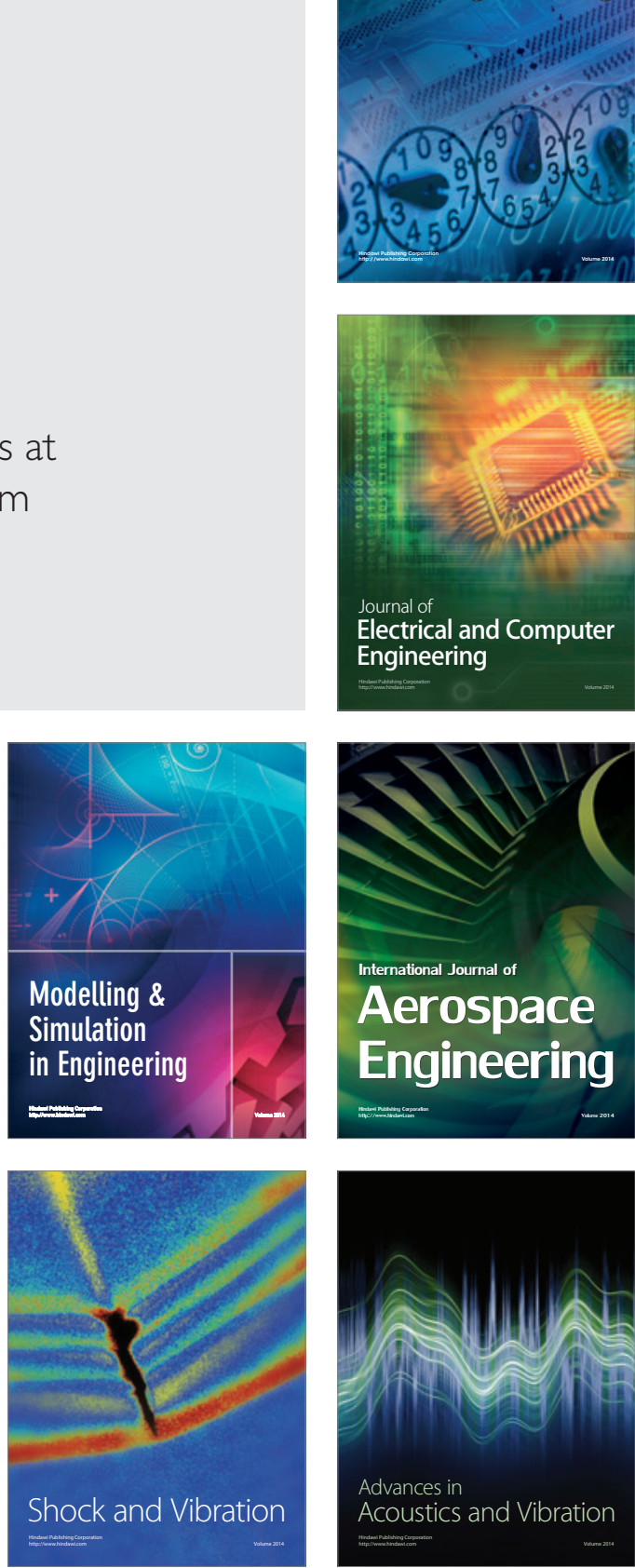